\title{
Observation on the Effect of Local Flap Transfer and Sclera Transplantation in Repairing Skin Defect after Eyelid Tumor Operation
}

\author{
Ying-Hui Ma ${ }^{1}$, Gang Liu ${ }^{2}$, Jie Yang ${ }^{1}$ \\ 1Department of Ophthalmology, the Affiliated Hospital of Chengde Medical University, Chengde, PR China. \\ 2Department of Ophthalmology, Southern Medical University, the Affiliated Fengxian Hospital, Shanghai, PR China.
}

\begin{abstract}
Introduction: Eyelid tumor refers to the primary eyelid skin and accessory tumors, which not only affects the appearance and vision, and even local infiltration and distant metastasis, thereby threatening life. Surgical resection is the most important and effective treatment, but tumor resection often leads to a wide range of eyelid defects, significantly affecting the shape and function of the eyelid. Therefore, the eyelid defect must be repaired after resection to achieve the purpose of aesthetic appearance and protection of the eyeball.

The objective of the study was to analyze the clinical results of local flap transfer and scleral transplantation in repairing skin defect after eyelid tumor operation.

Methods: Forty patients with postoperative skin defects of eyelid tumors admitted from December 2008 to December 2018 were taken for analysis and divided into two groups, 20 cases in each group. The control group underwent autologous lip mucous membrane transfer and nearby skin flap, while the experimental group received local skin flap rotation and scleral allograft repair. The clinical effect of repair of the two groups were compared. Results: In the experimental group, the effect of clinical repairs were better, the difference of height of palpebral fissure and the length of palpebral fissure were compared with the control group, which showed statistical significance favoring the experimental group.

Conclusion: Local flap transfer and scleral transplantation has better clinical outcome to repair the skin defect after operation of eyelid tumor.
\end{abstract}

Keywords: Local Flap Transfer, Scleral Transplantation, Eyelid Tumors, Skin Defect

\section{Introduction}

The eyelid is located on the outer surface of the eyeball and acts as a barrier to protect the eyeball. If the post excision defect of eyelid is not repaired effectively, the appearance of the eye and its visual function will be seriously affected. Anatomical and functional reconstruction of eyelid is necessary to establish an aesthetic look and for protection of the eyeball with ultimate goal functional conseravtion. The clinical data of 40 patients with eyelid tumor admitted to the Affiliated Hospital of Chengde Medical University and Southern Medical University
Affiliated Fengxian Hospital in last 10 years were retrospectively analyzed in this article.

\section{Methods}

From December 2008 to December 2018, 40 patients who underwent repair of eyelid skin defect after resection of tumor were selected from the department of ophthalmology of the Affiliated Hospital of Chengde Medical University and Southern Medical University Affiliated Fengxian Hospital. One group of 20 patients underwent autologous lip mucous membrane transfer

Correspondence

Gang Liu, Department of Ophthalmology, Southern Medical University Affiliated Fengxian Hospital, Shanghai, China.email:shliugang1976@163.com. 
and nearby skin flap. Another group of 20 patients ie, the experimental group received local skin flap rotation and scleral allograft repair. In group I, Male to female ratio was $11: 9$, maximum age was 72 , minimum age 38 , with median age of $(55.22 \pm 2.24)$. The male to female ratio in the experimental group was 12:8, the maximum age was 73 , the youngest age was 40 , with the median age of $54.36 \pm 3.27$.

Repair materials of the group I were obtained from autologous labial mucosa and adjacent skin flap. After the lower lip was everted, marking was done to draw the required size of lip mucosa. Full layer of lip mucosa was taken under local anaesthesia. Suitable skin flap was selected from the nasolabial or temporal side. In group II, local skin flap rotation and allograft scleral transplantation were performed. The size of the eyelid tumor was measured under the operating microscope (magnification was 3.5 times). The eyelid tumor was completely removed along the $2 \mathrm{~mm}$ margin around the tumor. In larger tumors, the whole of the eyelid was included, and the thin tissue rim of the cutting edge was taken for frozen section pathological examination to determine whether there were residual tumor cells at the margin of the surgery. In case of positive margins, the resection was extended again until the margin was negative. The width of the defect of eyelid under normal tension after tumor resection was measured under a surgical microscope. After the removal of tumor, the skin flap was designed according to the tissue defect. The pedicle width was $<2.0 \mathrm{~cm}$, and the full thickness skin and subcutaneous tissue were rotated. This flap is approximated in the defective area of the resection. The donor area was sutured after hemostasis of the wound taking care of the reduction of tension of the skin flap in consideration. This will prevent from the formation of cat eye deformity.

The sclera to be transplanted was be prepared form the homologous eyeball that was removed for end stage glaucoma or from the traumatic rupture of the eyeball. Sclera was harvested in the sterile environment, repeatedly rinsed with saline, which is then soaked in $95 \%$ ethanol solution for 3 days, and later stored in a sealed bottle of $75 \%$ ethanol at $-4^{\circ} \mathrm{C}$.
At the time of the transplant, the harvested sclera was irrigated with the saline for 10 minutes and pruned according to the size and characteristics of the eyelid defect, and the scleral film was placed toward the eyeball surface of the cornea. When repairing the upper eyelid of the patient, the eyelid defect wound was pruned as a rectangle, then a vertical curved incision was made on the skin of the inner and outer skin, and the orbicularis oculi muscle was sharply separated to fully expose the skin of the inner and outer skin. Then the transplanted sclera was fixed by suture at the skin position of the inner and outer skin. The upper edge of the levator palpebrae and sclera was sutured with 6-0 suture. When repairing the lower eyelid of the patient, the scleral slice was placed in the separated conjunctival sac, and the lower edge of the scleral slice and conjunctival membranes were sutured intermittently. After the operation, a loose bandage were applied for 24 to $48 \mathrm{~h}$.

Categories of the standards of repair: According to the curative effect standard, it was divided into four levels: 1.Excellent: eyelid appearance and complete recovery of function; 2. Good: eyelid shape and function were restored; incomplete closure $\leq 2 \mathrm{~mm}$; the difference in length of palpebral fissure $\leq 2 \mathrm{~mm}$; the difference in height $\leq 2 \mathrm{~mm}$; mild palpebral margin notch; 3 . Satisfactory: the appearance and function of the eyelids were improved, with incomplete closure $>2 \mathrm{~mm}$, the difference in length of palpebral fissure $>2 \mathrm{~mm}$, the difference in height $>$ $2 \mathrm{~mm}$. Eyelid margin notch, slight eyelid turning inside and outside; 4. Unsatisfactroy: eyelid appearance and function both were below satisfactory standards. Cure rate $=($ excellent + good $)$ cases $/$ total cases $\times 100 \%{ }^{1}$

Observational index: To observe and calculate the difference in clinical repair rate, height, and length of the palpebral fissure among the Group I (control group) and group II (experimental group). ${ }^{2}$

Statistical analysis: SPSS 22.0 statistical software was used for statistical analysis. The measurement data were expressed by $\mathrm{x} \pm \mathrm{s}$ and an independent sample $\mathrm{t}$-test was used. The counting information was expressed in numbers and percentages and rates were compared using the $\chi 2$ test, $\mathrm{p}<0.05$ was considered statistically significant. 
Table 1: Comparison of the cure rate of skin defect after operation of eyelid tumor between two groups

\begin{tabular}{|l|l|l|l|l|l|l|}
\hline \multicolumn{1}{|c|}{ Group } & \multicolumn{1}{|c|}{ n } & Excellent & Good & Satisfactory & Unsatisfactory & Total effective rate \\
\hline The experimental group II & 20 & 5 & 12 & 2 & 1 & $85 \%$ \\
\hline The control group I & 20 & 2 & 10 & 3 & 5 & $60 \%$ \\
\hline $\mathrm{x}^{2}$ & & & & & & 5.584 \\
\hline $\mathrm{p}$ & & & & & & 0.018 \\
\hline
\end{tabular}

As depicted in table 2, the difference between the height of palpebral fissure and the length of the palpebral fissure was lower in the experimental group than in the control group, and the difference was statistically significant $(\mathrm{p}<0.05)$.

Table 2: The difference in the height and length of the palpebral fissure between the two groups after eyelid surgery

\begin{tabular}{|l|l|l|l|}
\hline \multicolumn{1}{|c|}{ Group } & \multicolumn{1}{|c|}{$\mathbf{n}$} & \multicolumn{1}{|c|}{$\begin{array}{c}\text { The difference in the height of } \\
\text { palpebral fissure } \mathbf{( m m )}\end{array}$} & \multicolumn{1}{|c|}{$\begin{array}{c}\text { The length difference of palpe- } \\
\text { bral fissure }(\mathbf{m m})\end{array}$} \\
\hline The experimental group & 20 & $1.31 \pm 0.09$ & $1.45 \pm 0.04$ \\
\hline The control group & 20 & $1.83 \pm 0.11$ & $1.99 \pm 0.12$ \\
\hline $\mathrm{t}$ & & -15.926 & -15.987 \\
\hline $\mathrm{p}$ & & 0.000 & 0.000 \\
\hline
\end{tabular}

\section{Results}

The cure rate of postoperative skin defect of tumor of eyelid in the experimental group was higher than that in the control group, the difference was statistically significant $(\mathrm{p}<0.05)$, as shown in table 1 .

\section{Discussion}

The human eye has a unique physiological function of seeing, which also expresses the human emotion and appearance. Eyelid has a center position in functionality of covering eyeball as well as in expressing the emotions. Even though it looks simple protective barrier of the eyeball, the imperfect repair after the surgical resection will result in functional difficulties of eye vision as well as of the psychological issues in the person. So perfect cosmetic as well as functional surgical repair is necessary. ${ }^{3}$

Eyelid skin can have different diseases that give rise to tumor. The prognosis depends on the tissue diagnosis of disease, unclear margins on surgical excision. The basic principle of surgery is to completely remove the tumor in the premise of the maximum retention of the healthy eyelid tissue, to provide a good foundation for eyelid reconstruction. First choice of treatment of Eyelid tumor is surgical excision and repair/reconstruction of the eyelid as soon as possible. ${ }^{4} \mathrm{With}$ the improvement of living standard and medical technology in recent years, people pay more and more attention to appearance, which also raises the relevant standard requirements of traditional eye surgery.

In the past, in the selection of palpebral substitutes, autologous labial mucosa or adjacent local flaps were the mostly used replace. This type of repair has certain cosmetic repair effects but causes trauma and pain at donor site in the mouth. ${ }^{5}$ Labial mucousal epithelium belongs to the non-keratinized epithelium and consists of two layers of cells. The dense connective tissue of the subepithelial lamina propria is thin, and the dermis and collagen fibers are thin and disordered. The connective tissue papilla are short and irregular and contains less elastic fibers, so it is too soft and elastic. It lacks toughness and necessary support, and is easily broken. At the same time, the low rate of uptake after grafting was also one of the factors leading to poor postoperative results. Other issues are postoperative contraction of graft was prone to eyelid varus, eyelid ectropion, eyelid atrophy, deformation, and other conditions, affecting the appearance, and in severe cases might affect eye function. ${ }^{6}$

The allograft sclera is an irregular dense connective tissue mainly composed of collagen fibers. The thick collagen 
fibers interweave into a dense lamellar structure, and there are only a few matrix synthetic fiber cells between the fibers. This structure determines the low antigenicity of the sclera. After preservation, its remaining fibers and amorphous matrix were even less able to elicit an immune response. The clinical observation showed that the reaction caused by scleral transplantation was slight. The scleral graft can induce the generation of receptor connective tissue while dissolving, which is an ideal material to replace the inner palpebral lining. The allogeneic scleral material was extracted from the eyeball of HBsAg negative patients without malignant tumors and inflammation. The allogeneic sclera is the best biomaterial for palpebral defect repair. After allogeneic scleral transplantation, the final result is replaced by connective tissue, which only plays the role of the scaffold, which not only meets the requirements of eyelid function but also reduces the pain. The local sliding skin flap, rotating skin flap, or free skin flap were selected to repair the eyelid defect according to the location, degree, and skin relaxation of the patient.

\section{Conclusion}

The eyelid defect caused by surgery in patients necessitate a perfect cosmetic as well as functional closure. Among reconstruction methods, flexible application of biological material such as allogeneic sclera and all kinds of skin flap transfer method to repair the defect is one of the method that is better compared to lower lip skin graft which can effectively restore the structure of the eyelid aesthetically and functionally. This method is worthy of clinical popularization and application.

\section{References}

1. Jiang $\mathrm{H}$, Jin CL. Application of local flap in repairing the defect after resection of malignant skin tumor. Jilin Medical Journal. 2018, 39(5):931-932.

2. Cheng X, Yang QF, Mu XX, et al. Clinical application of tarso-conjunctival flap in reconstruction of posterior eyelid defects of eyelid tumors. Chin J Plast Surg. 2018, 34(6):458-463.

3. O,Donnell BA, Candenmir OO. Maximal eyelid donor skin harvesting in eyelid repair after tumor excision. Ophthal Plast Reconstr Surg. 2002, 18(6) :436-440.

4. Dias FC, Danielski A, Forster K, et al. Use of a subdermal plexus flap to reconstruct an upper eyelid following radical tumor resection in a cat. J Am Vet Med Assoc. 2017, 250(2):211-214.

5. Hishmi AM, Koch KR, Matthaei M, et al. Modified Hughes procedure for reconstruction of large fullthickness lower eyelid defects following tumor resection. Eur J Med Res. 2016, 21(1):27.

6. Kopecky A, Koch KR, Bucher F, et al. Results of CutlerBeard procedure for reconstruction of extensive full thickness upper eyelid defects following tumor resection. Ophthalmologe. 2016, 113(4):309-313.

7. Codner MA, McCord CD, Mejia JD, et a1. Upper and lower eyelid reconstruction. PLast Reconstr Surg. 2010,126(5):231e-245e. 